\title{
Distance Transforms in Multi Channel MR Image Registration
}

\author{
Min Chen ${ }^{1}$, Aaron Carass $^{1}$, John Bogovic ${ }^{1}$, Pierre-Louis Bazin ${ }^{2}$ and Jerry L. Prince ${ }^{1}$ \\ ${ }^{1}$ Image Analysis and Communications Laboratory, \\ ${ }^{2}$ The Laboratory of Medical Image Computing, \\ The Johns Hopkins University, Baltimore, MD 21218
}

\begin{abstract}
Deformable registration techniques play vital roles in a variety of medical imaging tasks such as image fusion, segmentation, and post-operative surgery assessment. In recent years, mutual information has become one of the most widely used similarity metrics for medical image registration algorithms. Unfortunately, as a matching criteria, mutual information loses much of its effectiveness when there is poor statistical consistency and a lack of structure. This is especially true in areas of images where the intensity is homogeneous and information is sparse. Here we present a method designed to address this problem by integrating distance transforms of anatomical segmentations as part of a multi-channel mutual information framework within the registration algorithm. Our method was tested by registering real MR brain data and comparing the segmentation of the results against that of the target. Our analysis showed that by integrating distance transforms of the the white matter segmentation into the registration, the overall segmentation of the registration result was closer to the target than when the distance transform was not used.
\end{abstract}

Keywords: Image registration, Magnetic resonance imaging, Multidimensional signal processing, Spatial normalization, Distance Transform.

\section{INTRODUCTION}

Deformable image registration is a crucial tool in medical image analysis, with applications including the characterization of anatomical variability, surgical planning, and atlas based segmentation. ${ }^{1-3}$ All of these applications are driven by the assumption that medical images can be deformably registered into a common frame of reference, thus providing spatial alignment. There has been extensive work on deformable image registration over the years, ${ }^{4-9}$ and the current literature offer a large variety of algorithms, each with their own approach for performing the optimization and deformation necessary for image alignment.

The general framework of a deformable registration problem takes a source image $\mathcal{S}$ and attempts to map it to a target image $\mathcal{T}$ through some deformation $v: \mathcal{D}_{\mathcal{S}} \rightarrow \mathcal{D}_{\mathcal{T}}$, where $\mathcal{D}$ is the domain of the respective images. One common factor among all existing registration algorithm is the use of a similarity metric, often incorporated into an energy function, to determine how close a given mapping brings the source to the target image. In recent years, mutual information, originally suggested by Viola and Wells, ${ }^{7}$ has become one of the most prominent similarity metrics used in medical image registration, with widespread presence in both academic and clinical applications. ${ }^{6}$

The strength of mutual information comes primarily from its lack of dependence on explicit intensity relationships between the images. As a result it is highly robust and applicable for a large number of image modalities. Unfortunately, despite its many advantages in matching mono- and multi-modal images, there are still several major drawbacks that affects its performance.$^{6,9,10}$ One of these drawbacks is its loss of effectiveness as a matching criterion when working within areas of an image that has poor statistical consistency and a lack of clear structure. ${ }^{11}$ Examples of this includes cases where there is overwhelming noise or conversely, when the area has very homogeneous intensity and provide very little information. The intent of this paper is to present a method that address the latter issue by using a distance transform of anatomical segmentations to provide relevant image features in these areas that lack intensity information.

Further author information, send correspondence to Jerry L. Prince:

E-mail: prince@jhu.edu, Telephone: +1-410-516-5192

Medical Imaging 2011: Image Processing, edited by Benoit M. Dawant, David R. Haynor,

Proc. of SPIE Vol. 7962, 79621D - @ 2011 SPIE · CCC code: 1605-7422/11/\$18 - doi: 10.1117/12.878367

Proc. of SPIE Vol. 7962 79621D-1 


\section{METHODS}

\subsection{Mutual Information}

For a discrete random variable $\mathcal{A}$, with $P_{\mathcal{A}}(a)$ representing the probability of the value $a$ occurring in $\mathcal{A}$, the Shannon Entropy ${ }^{12}$ is defined by $H(\mathcal{A})=-\sum_{a} P_{\mathcal{A}}(a) \log \left(P_{\mathcal{A}}(a)\right)$. Similarly, for a second random variable $\mathcal{C}$ and joint probability distribution $P_{\mathcal{A}, \mathcal{C}}(a, c)$, the joint entropy is $H(\mathcal{A}, \mathcal{C})=-\sum_{a, c} P_{\mathcal{A}, \mathcal{C}}(a, c) \log \left(P_{\mathcal{A}, \mathcal{C}}(a, c)\right)$. If the random variable represents image intensity values, its entropy measures how well a given intensity value in an image (or a pair of intensities in two images) can be predicted. Using this, the mutual information of two images being registered is defined as:

$$
\mathrm{I}(\mathcal{T}, v(\mathcal{S}))=H(\mathcal{T})+H(v(\mathcal{S}))-H(\mathcal{T}, v(\mathcal{S}))
$$

where $v(x)$ represents the deformation between a source image $\mathcal{S}$ and a target image $\mathcal{T}$, and the mutual information is maximized when $v(x)$ maps $\mathcal{S}$ exactly to $\mathcal{T}$. Frequently, normalized mutual information (NMI), ${ }^{13}$

$$
\operatorname{NMI}(\mathcal{T}, v(\mathcal{S}))=\frac{H(\mathcal{T})+H(v(\mathcal{S}))}{H(\mathcal{T}, v(\mathcal{S}))}
$$

is used in place of mutual information and has been shown to be a more robust and consistent measure.

\subsection{Drawback of Mutual Information}

One of the primary issues with using mutual information for image comparison is its poor response to areas where the image is homogeneous. Take for example a patch of an image where there is only a single intensity. In this case the total entropy of that patch is zero, and the joint entropy with any other patch is equivalent to the entropy of the compared patch. Hence, in this case the mutual information is always zero regardless of what the homogeneous patch is compared to. This causes algorithms that use local optimization to maximize mutual information to have problems when directing the deformation of these areas, since they can be effectively matched with any other patch. As a result, this makes these areas less reliable in the final registration.

This problem can be best demonstrated when using mutual information to register two binary images, as shown in Figure 1. The example shows the registration between two white matter masks, which, due to the lack of intensity information present, produced poor alignment and overall noisy boundaries in the registration result. However, when the same registration was performed on the the distance transform of the segmentation, the alignment was significantly sharper and incorporated finer details. In this case, the distance transform of the segmentation populated the image with strong structural features that allowed mutual information to find a more accurate alignment.

\subsection{Registration}

The registration algorithm used in our experiments was an adaptation of the Adaptive Bases Algorithm(ABA). The algorithm uses mutual information to approximate the total deformation $v(x)$ by a summation of radial basis functions (RBFs), $\Phi(x)$ :

$$
v(x)=\sum_{i} w_{i} \Phi\left(\left\|x-c_{i}\right\|\right)
$$

where $c_{i}$ are the center locations of the RBFs and $w_{i}$ are the coefficients being optimized. This method was chosen primarily for its ability to provide homeomorphic deformations while allowing each individual RBF to be optimized independently, therefore increasing its speed.

\subsection{Integrating Distance Transform of Anatomical Segmentation}

The main contribution of our method comes from the application of distance transforms to assist registration algorithms in areas that lack intensity information. For our experiments, we identified three areas in brain magnetic resonance (MR) images where this is true: the white matter, the gray matter, and the combination of the putamen and caudate. The segmentations for these areas were performed by using the TOpology-preserving, 

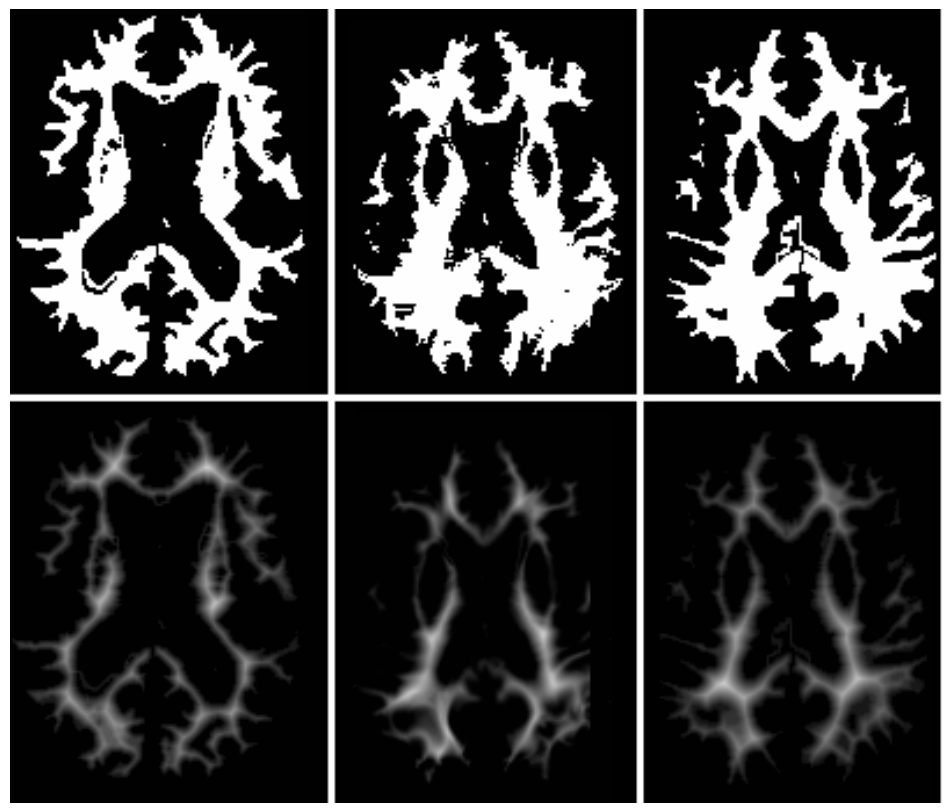

Source

Result

Target

Figure 1: Registration results between two white matter segmentation masks(top row) and the same registration between their distance transforms(bottom row).

Anatomy-Driven Segmentation (TOADS) ${ }^{14,15}$ algorithm, which is capable of automatically producing topologically accurate segmentation of these structures.

TOADS was chosen for its high level of accuracy and robustness when compared to manual segmentation of cortical structures, and for its ability to automatically produce topologically correct results. The latter is particularly important when performing distance transforms, since small topology defects within the segmentation, such as a hole or handle, can produce relatively large artifacts in its distance transform. Once the segmentation was produced, its distance transform was calculated, where every point inside the segmentation is mapped as its minimum distance to the boundary of the segmentation.

The additional information provided by the distance transform was integrated into the mutual information framework using a weighted multi-channel adaptation of ABA. ${ }^{16}$ In this approach, the original source and target images were treated as one channel, and their distance transforms were treated as a second channel. The registration for both channels were done simultaneously by optimizing a single deformation. However, the energy function used in the optimization was calculated as a weighted summation of the NMI of each channel separately:

$$
\mathrm{E}=\sum_{k} g_{k} \operatorname{NMI}\left(\mathcal{T}_{k}(\alpha), v\left(\mathcal{S}_{k}(\alpha)\right)\right)
$$

where $k$ specified the channel, and $g_{k}$ was the channel weight. For the purpose of this study, the weights were treated as equal for both channels. Since the distance transform overlaps the white matter directly, this formulation allowed it to to drive the registration in those areas where the image was too homogeneous for mutual information to be effective.

\subsection{Data}

The data used to evaluate our method consisted of 20 magnetization-prepared, rapid gradient echo (MPRAGE) MR images of the brain from the OASIS data set (http://www.oasis-brains.org/). The images were skull stripped using the SPECTRE ${ }^{17}$ algorithm. 


\section{RESULTS}

\subsection{Mutual Information With Regard to Rotation}

To observe the behavior of mutual information when using an intensity, mask or distance transform image, we plotted the change in mutual information between each image and itself rotated in 5 degree increments. Figure 2 shows these plots when using a MR brain image, and its white matter mask and distance transform. From the plots we can see that while all three images maintained the same overall trend, the range and rate of change differed significantly. In particular, the distance transform produced a much higher maximum and a smoother drop off when compared to the WM mask. These two qualities play an important role in optimization, since a larger maximum makes it easier to avoid local extremas, and smooth transitions into the maximum provides an easier search space. This is particularly true for algorithms that use gradient descent type methods for optimization. The MR image of the whole brain provided the best characteristics for optimization, since it contained the strongest features and the most information.

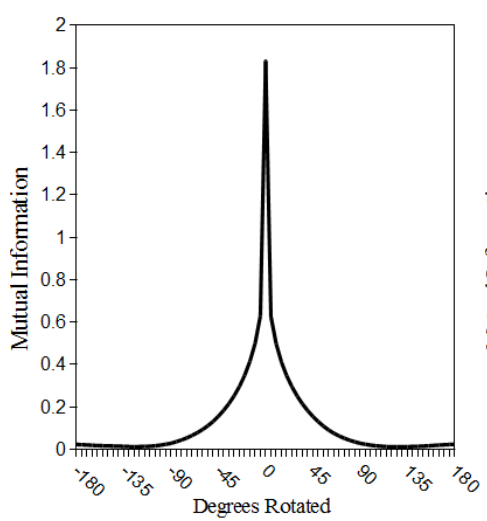

(a) MR Whole Brain

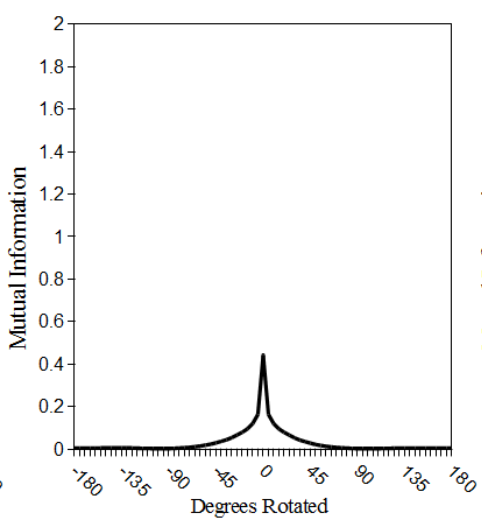

(b) White Matter Mask

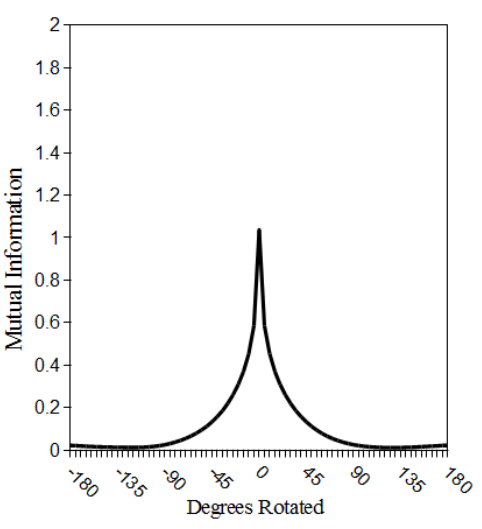

(c) WM Distance Transform

Figure 2: Plots of mutual information as a function of rotation for an MR brain image, its white matter mask and distance transform.

\subsection{Single-Channel Known Deformation Recovery}

Table 1 shows the average absolute error from a known deformation recovery experiment using brain MR images and their respective white matter masks and distance transforms. For this experiment, a known deformation was applied to an image, and the deformed image was registered to the original image to recover the deformation. The absolute error between the recovered and known deformations were then calculated and averaged to measure the accuracy of the registration. This process was repeated and averaged over 10 different OASIS brain images. To prevent bias towards images with larger volumes, the error was only calculated over the union of the nonbackground voxels in the source and target images for each case. The applied known deformation was a three dimensional sinusoid ranging from -9 to 9 voxels displacement.

\begin{tabular}{|c|c|c|c|}
\hline & $\begin{array}{c}\text { MR Whole } \\
\text { Brain }\end{array}$ & $\begin{array}{c}\text { White Matter } \\
\text { Mask }\end{array}$ & $\begin{array}{c}\text { WM Distance } \\
\text { Transform }\end{array}$ \\
\hline Average Error(Voxels) & 1.07 & 2.94 & 1.09 \\
\hline
\end{tabular}

Table 1: Average error of the recovered deformation field, when using the whole MR image, the white matter mask, and the distance transform of the white matter mask.

The result show that the white matter mask performed significantly worse for recovering the known deformation when compared to the distance transform or MR image. The primary reason for this is due to a lack of features in the white matter mask that would allow various parts of the image to be differentiated. Since the entire mask is one intensity, a voxel can be deformed from almost any direction in order to match with the target. 


\subsection{Segmentation Comparison of Multi-Channel Results}

To evaluate the performance of our multi-channel approach, 10 separate registrations were performed using different pairs of source and target brain MR images. Each registration result was then segmented by TOADS, and the overlap between the result segmentation and the target image segmentation was considered using Dice's coefficient, $\operatorname{Dice}(A, B)=\frac{2|A \cap B|}{|A|+|B|}$. This value ranges from zero to one, with one indicating a perfect overlap. Table 2 shows the Dice's coefficient between various structures from the segmentations. This experiment was repeated using just the MR images, the white matter mask as a second channel, and the white matter mask distance transform as a second channel.

From the results, we see that using the distance transform as a second channel improved the overall overlap for the resulting anatomical segmentations. Figure 3 shows an example of this result, where we can see that using the distance transform allowed the white matter boundary to be much more sharply aligned. However, from the table we also see that using the white matter mask as the second channel significantly decreased the performance of the algorithm. This result was as expected, since the lack of intensity information in the white matter is the problem being addressed by our method. Adding the white matter mask as a second channel elevated this problem by effectively weighting the influence of these areas even more.

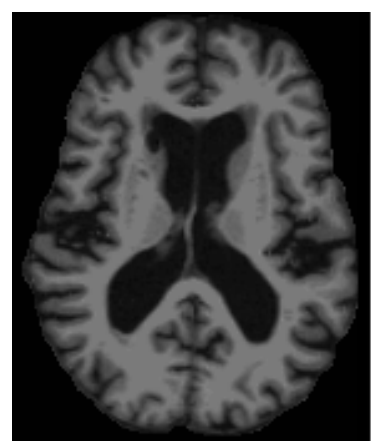

Source

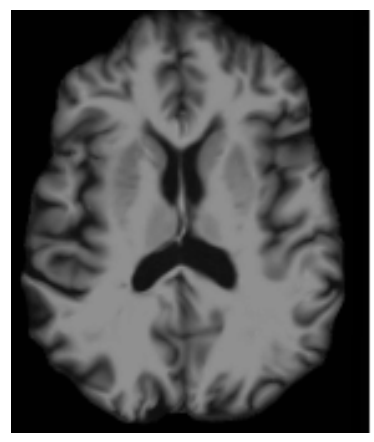

(a)

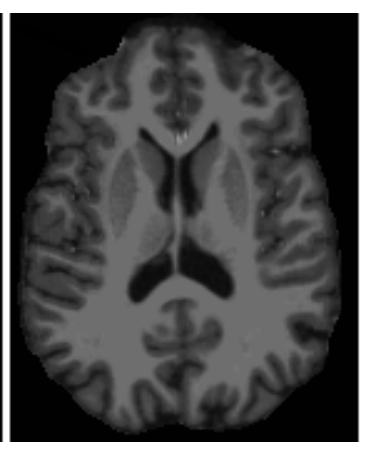

Target

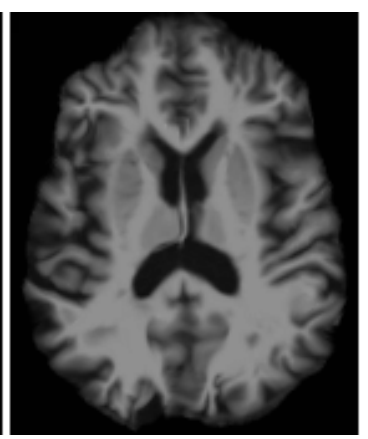

(b)

Figure 3: Single and multi-channel registration results between a source and target image. (a) shows the result when only using the MR images and (b) shows the result when using the distance transform of the white matter segmentation as a second channel.

\section{DISCUSSION AND CONCLUSION}

In this paper we have presented a novel integration of distance transforms of anatomical segmentation to assist mutual information based registrations in areas of images that lack significant intensity information. We have shown how homogeneous regions can negatively impact the effectiveness of mutual information as a similarity metric when recovering a known deformation. In addition, our results show that our adaptations improved the 


\begin{tabular}{|c|c|c|c|}
\hline Region & MR Image Only & $\begin{array}{c}\text { MR Image + WM } \\
\text { Mask }\end{array}$ & $\begin{array}{c}\text { MR Image + WM } \\
\text { Distance Transform }\end{array}$ \\
\hline Grey Matter & .759 & $.603(-20.6 \%)$ & $.770(+1.45 \%)$ \\
White Matter & .830 & $.640(-22.9 \%)$ & $.841(+1.33 \%)$ \\
Putamen and Caudate & .804 & $.671(-16.5 \%)$ & $.830(+3.23 \%)$ \\
\hline
\end{tabular}

Table 2: Overlap comparison using Dice's coefficient between segmentations of the registration result and segmentations of the target image for various brain regions, averaged over 10 registrations. The three columns represent(from left to right) when the registration was performed with just a the MR image, and when the white matter mask and its distance transform were used as second channels. Shown in parentheses are the percentage increase or decrease from adding the second channel.

accuracy of MR brain registrations when comparing the segmentation of the results to the segmentation of the target image. This suggests that our method is capable of improving registration in areas of the image where there is not enough information provided for mutual information to effectively align the source image to the target image. The method described in this paper, along with TOADS and SPECTRE are freely available for download as part of the Java Imaging Science Toolkit (JIST, http://www.nitrc.org/projects/jist/). ${ }^{18,19}$

\section{ACKNOWLEDGEMENT}

This work was supported in part by NIH/NINDS grants K25DA025356, R01NS056307, and R01NS070906.

\section{REFERENCES}

[1] Goldszal, A. F., Davatzikos, C., Pham, D. L., et. al., "An image processing system for the qualitative and quantitative volumetric analysis of brain images," J. Computer Assisted Tomography 22, 827-837 (1998).

[2] Gur, R. C., Mozley, P. D. and Resnick, S. M., "Gender deifferences in age effect on brain atrophy measured by magnetic resonance imaging," Proc. Natl. Acad. Sci. 88, 2845-2849 (1991).

[3] Resnick, S. M., Goldszal, A. F., Davatzikos, C., et. al., "One-year age changes in MRI brain volumes in older adults," Cerebral Cortex 10(5), 464-472 (2000).

[4] Rohde, G. K., Aldroubi A. and Dawant, B. M., "The Adaptive Bases Algorithm for intensity based nonrigid Image Registration," IEEE Trans. Med. Imag. 22(11), 1470-1479 (2003).

[5] Rohr, K., Stiehl, H. S., Sprengel, R., et. al., "Landmark-base elastic registration using approximating thin-plate splines," IEEE Trans. Med. Imag. 20(6), 526-534 (2001).

[6] Pluim, J. P. W., Maintz, J. B. A. and Viergever, M. A., "Mutual Information Based Registration of Medical Images: A Survey," IEEE Trans. Med. Imag. 22(8), 986-1004 (2003).

[7] Wells III, W. M., Viola, P., Atsumi, H., Nakajima, S. and Kikinis, R., "Multimodal volume registration by maximation of mutual information," Medical Image Analysis 1(1), 35-51 (1996).

[8] Shen, D. and Davatzikos, C., "HAMMER: Hierarchical Attribute Matching Mechanism for Elastic Registration," IEEE Trans. Med. Imag. 21(11), 977-1000 (2002).

[9] Maes, F., Collignon, A., Vandermeulen, D., Marchal, G. and Suetens, P., "Multimodality image registration by maximization of mutual information," IEEE Trans. Med. Imag. 16(2), 187-198 (1997).

[10] Likar, B. and Pernus, F., "A hierarchical approach to elastic registration based on mutual information," Image Vision Comput. 19, 33-44 (2001).

[11] Andronache, A., Siebenthal, M. von, Szkely, G. and Cattin, Ph., "Non-rigid registration of multi-modal images using both mutual information and cross-correlation," Medical Image Analysis 5(1), 3-15 (2008).

[12] Shannon, C. E., "A Mathematical Theory of Communication," Bell System Technical Journal 27, 379-423 (1948).

[13] Studholme, C., Hill, D. L. G. and Hawkes, D. J., "An overlap invariant entropy measure of 3D medical image alignment," Pattern Recognition 32(1), 71-86 (1999).

[14] Bazin, P.-L. and Pham, D. L., "Topology-preserving tissue classification of magnetic resonance brain images," IEEE Trans. Med. Imag. 26(4), 487-96 (2007).

[15] Bazin, P.-L. and Pham, D. L., "Homeomorphic brain image segmentation with topological and statistical atlases," Medical Image Analysis 12, 616-625 (2008).

[16] Chen, M., Carass, A., Wheeler, B., Liu, X. and Prince, J. L., "Multi-channel enhancement of the adaptive bases algorithm," in [16th Annual Meeting of the Organization for Human Brain Mapping], (2010). 
[17] Carass, A., Wheeler, M. B., Cuzzocre, J., Bazin, P.-L., Bassett, S. S. and Prince, J. L., "A joint registration and segmentation approach to skull stripping," in [Fourth IEEE International Symposium on Biomedical Imaging (ISBI 2007)], 656-659 (2007).

[18] Lucas, B. C., Bogovic, J. A., Carass, A., Bazin, P. L., Prince, J. L., Pham, D. L., and Landman, B. A., "The java image science toolkit (jist) for rapid prototyping and publishing of neuroimaging software," Neuroinformatics $\mathbf{8}(1)$, 5-17 (2010).

[19] Landman, B. A., Lucas B. C., Carass A., Prince J. L., "A rapid prototyping environment for neuroimaging in java," in [15th Annual Meeting of the Organization for Human Brain Mapping], (2009).

Proc. of SPIE Vol. 7962 79621D-7 